\title{
Analysis and Nonlinear Simulation of a Quadrupole Wiggler FEL at Millimeter Wavelengths
}

\author{
SHENG-FUH CHANG, OWEN C. ELDRIDGE AND JOHN E. SCHARER
}

\begin{abstract}
We examine the use of a helical quadrupole wiggler field in a circular guide for amplification of millimeter wavelength waves. The effects of space charge and axial guide field are considered for beam stability for near-axis orbits. When space charge is neglected, we examine the orbit equations and properties and characteristic betatron oscillation frequencies by a linearization of the orbit equations. A nonlinear simulation code developed by NRL for dipole wigglers is modified to examine optimum gain $\mathrm{TE}_{21}$ waveguide modes for a quadrupole wiggler in the 30-300 $\mathrm{GHz}$ range. Particle orbit trajectories well offaxis for an annular beam are examined, as well as nonlinear gain, efficiency at saturation, and characteristic betatron oscillations, illustrating classic quadrupole focusing effects for an untapered wiggler. Finally, the quadrupole wiggler is compared to an equivalent dipole wiggler case to illustrate the advantages and drawbacks of each case.
\end{abstract}

\section{INTRODUCTION}

$\mathrm{W}$ E consider a free-electron laser (FEL) with a helical quadrupole magnetic wiggler field inside a circular guide in the millimeter-wave range. The quadrupole was chosen because its known focusing and stability properties for accelerators might provide improvements at high power and beam density where space charge effects and particle orbit trajectories could be stabilized. A tunable FEL in the millimeter-wave range has important applications in the areas of communications, high-resolution radar, semiconductor and solid-state material properties measurements, and as a source for heating fusion plasmas. Earlier work by Levush et al. [1] examined the nearaxis orbit properties of a quadrupole wiggler without an axial guide field but including the effects of space charge and showed that it had improved beam stability when compared to a dipole wiggler. Work by Ganguly and Freund [2] developed a nonlinear simulation code for a helical dipole wiggler FEL to examine optimized circular waveguide mode, particle orbits, gain and efficiency. We have modified this code to examine the properties of a helical quadrupole wiggler. Our preliminary work on the quadrupole FEL [3] at $280 \mathrm{GHz}$ has shown high gain, broad bandwidth and moderate efficiency for an untapered wiggler. In this paper we examine the off-axis orbit equations to obtain the betatron frequency as well as the quadrupole focusing properties, optimized gain, efficiency, and bandwidth at $33 \mathrm{GHz}$, and a comparison with an equivalent dipole wiggler configuration.

Manuscript received June 14, 1988. This work was supported in part by the Research Committee of the University of Wisconsin and by the $\mathrm{Na}$ tional Science Foundation under Grant ECS-85-14978.

The authors are with the Department of Electrical and Computer Engineering, University of Wisconsin, Madison, WI 53706.

IEEE Log Number 8823269.
Experiments using helical dipole wigglers and guide fields have been recently carried out by Fajans et al. [4][6] and Pershing et al. [7] who measured the nonlinear radiation intensity and phase in the Raman regime at 9.3 $\mathrm{GHz}$, verifying the theoretical predictions. The same NRL simulation code (ARACHNE) [2] that we have modified was used to model this experiment, and obtained good agreement with the experiment at a power level of 100 $\mathrm{kW}$ with 10 percent efficiency.

Research on quadrupole FEL's without an axial guide field has been recently carried out jointly by the Maryland and NRL research groups. The first work by Levush et al. [1] examined near-axis orbit stability including high current density solid beam collective space-charge effects, and found that high currents approaching the AlfvenLawson limit can be stably injected into a quadrupole wiggler. The gain was evaluated for a plane wave with no radial structure and weak wiggler field strengths. Regions of positive gain were found which had the same functional wiggler field strength dependence as the dipole pump. In a later paper Levush et al. [8] generalized these results to larger values of wiggler field strength by utilizing the Madey theorem. This theorem calculates the gain as the energy derivative of the spontaneous emission transition rate into the mode excited by the incident stimulating radiation. They found gain at higher values of the quadrupole wiggler field which allows the FEL oscillator to operate at two, three, or four frequencies by tuning the cavity to the appropriate "betatron frequency."

More recent work by Antonsen and Levush [9] examined the nonlinear theory of a quadrupole free-electron laser when the betatron frequency is close to the mismatch frequency and reduced the three-dimensional equations to an integrable one-dimensional equation. They consider the effect of a taper and obtain conditions for electrons to remain trapped in the ponderomotive well as the beat wave decelerates. This theory can be used to design a taper and evaluate the enhanced efficiency in a full nonlinear code.

These theories for the rotating quadrupole wiggler FEL all assume a plane wave signal field, which approximates the central region of a $\mathrm{TE}_{11}$ mode. We find in the nonlinear simulation that the $\mathrm{TE}_{21}$ mode is dominant.

\section{Analysis of the Helical Quadrupole FEL}

We have begun a program for the analysis of the helical quadrupole FEL so that we may compare this configuration to alternative configurations, determine the influence 
of design parameters on FEL performance, and establish benchmarks for checking the nonlinear computer simulation. We have accomplished several tasks in this analysis. First we have examined the stability to betatron oscillations in the configuration. This analysis goes beyond the work by Levush et al. [1], [8]. We include an axial magnetic field that provides an additional parameter for optimizing the gain, bandwidth, and stability. We can also predict the frequency for the peak of the gain and efficiency curves from the simulation of this FEL configuration, as a function of the FEL parameters. Although the device has a very broad bandwidth, this relation is very important for the design and development of this FEL.

Fig. 1 illustrates schematically the quadrupole wiggler configuration. The conductors are wound on the guide tube or a mandrel in a spiral pattern, with currents alternating in direction. The quadrupole wiggler can be produced by the currents as shown or an array of permanent magnets on the circular guide which has the advantage of no ohmic dissipation.

\section{A. Orbits in a Linearized Quadrupole Wiggler and Axial Field}

A vector potential solution of the static Maxwell equations in vacuum for a helical quadrupole wiggler field and a uniform guide field is

$$
\begin{aligned}
& A_{r}=-\left(B_{w} / k_{w}^{2} r\right) I_{2}(2 \lambda) \cos (2 \chi) \\
& A_{\theta}=B_{o} r / 2+\left(B_{w} / k_{w}\right) I_{2}^{\prime}(2 \lambda) \sin (2 \chi)
\end{aligned}
$$

where $I_{2}$ is the Bessel function of imaginary argument and $I_{2}^{\prime}$ is its derivative. Cylindrical coordinates $(r, \theta, z)$ are used, with

$$
\begin{aligned}
& \chi=\theta-k_{w} z \\
& \lambda=k_{w} r
\end{aligned}
$$

and

$$
k_{w}=2 \pi / \lambda_{w} .
$$

The static magnetic fields are

$$
\begin{aligned}
& B_{r}=2 B_{w} I_{2}^{\prime}(2 \lambda) \cos (2 \chi) \\
& B_{\theta}=-\left(2 B_{w} / \lambda\right) I_{2}(2 \lambda) \sin (2 \chi)
\end{aligned}
$$

and

$$
B_{z}=B_{o}+2 B_{w} I_{2}(2 \lambda) \sin (2 \chi) .
$$

These fields have the form of a right-handed screw in space. Radial plots of these fields are shown in Fig. 2 . The wiggler field goes to zero at $r=0$.

To characterize the magnitude of the wiggler field, we will usually give the average magnetic field $B_{w}\left(r_{g}\right)$ at the radius of the circular guide tube $r_{g}$. This field is related to $B_{w}$ through the relation

$$
\begin{aligned}
B_{w}\left(r_{g}\right)= & B_{w}\left[I_{1}\left(2 k_{w} r_{g}\right)^{2}+2 I_{2}\left(2 k_{w} r_{g}\right)^{2}\right. \\
& \left.+I_{3}\left(2 k_{w} r_{g}\right)^{2}\right]^{1 / 2} .
\end{aligned}
$$

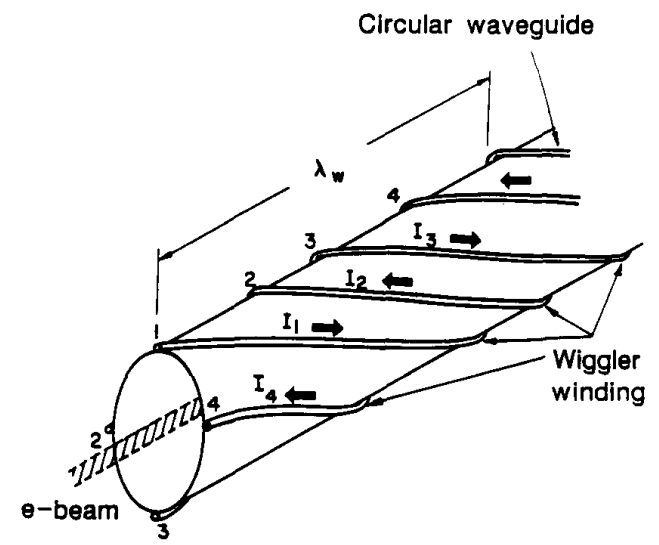

Fig. 1. An FEL configuration with a quadrupole wiggler.

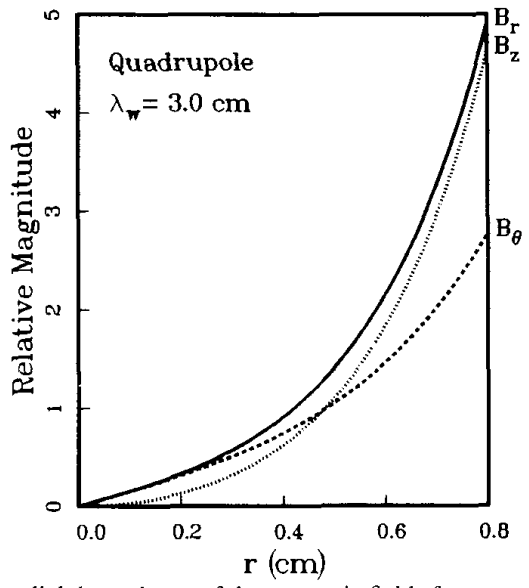

Fig. 2. The radial dependence of the magnetic fields for a quadrupole wiggler.

These fields are the lowest terms in a multipole expansion for a helical quadrupole current configuration and are not a particular solution for given currents and are not valid near the currents.

The FEL is driven by the transverse (betatron) oscillations of the relativistic beam in the wiggler field $B_{w}$ and the axial field $B_{o}$. This calculation gives the motion of the electrons in the beam before the signal field builds up to appreciable amplitude. Of primary interest is the stability of the beam, the frequencies of the betatron oscillations, and the structure of the orbits, which are needed for the calculation of the gain and efficiency.

In this calculation we also include the self-fields of the unneutralized electron beam produced by the charge density and current density, for a cold cylindrical beam with uniform cross sections. The charge and pinch forces, taken alone, produce a force that is always repulsive, so that the beam is unstable and strikes the wall. The addition of the quadrupole wiggler field $B_{w}$ will stabilize the beam for a range of wiggler field strengths, as shown by Levush et al. [1]. Here we include both the helical quadrupole wiggler field and the axial field; the additional parameter produces an added flexibility for insuring beam stability, and optimizing the gain and efficiency of the FEL. 
To solve the equations of motion analytically, we expand the complete wiggler fields given by (1) in powers of the radius. The expansion is valid for $k_{w} r<1$, or essentially out to half the waveguide radius. This expansion is adequate for the regime in which the signal fields may be neglected. This expansion is used for analytic tractability, and is not used in the simulation. In Cartesian coordinates, these fields are

$$
\begin{aligned}
& B_{\chi} \approx B_{w} k_{w}[x \cos (2 \phi)+y \sin (2 \phi)] \\
& B_{y} \approx B_{w} k_{w}[-y \cos (2 \phi)+x \sin (2 \phi)]
\end{aligned}
$$

and

$$
B_{z} \approx B_{o}
$$

where

$$
\phi=k_{w} z
$$

\section{B. Space Charge}

A uniform cold cylindrical beam with radius $r_{b}$, with electrons of axial velocity $v_{z o}$ and density $n_{o}$, carries a current

$$
I_{b}=-e n_{o} v_{z o} \pi r_{b}^{2}
$$

The space charge of the unneutralized electron beam produces a radial electric field

$$
E_{r}=-2 \pi e n_{o} r
$$

and the current produces an azimuthal magnetic field

$$
B_{\theta}=-2 \pi e n_{o} v_{z o} r / c \text {. }
$$

The resultant of the repulsive radial space charge and the attractive magnetic pinch is the radial force

$$
F_{r}=2 \pi e^{2} n_{o} r\left(1-v_{z o}^{2} / c^{2}\right) \approx 2 \pi e^{2} n_{o} r / \gamma^{2}
$$

when the transverse velocity is much smaller than the axial velocity. The combination of these forces is destabilizing.

\section{Betatron Oscillations}

The equations of motion in these fields are

$$
\begin{aligned}
d v_{x} / d t \approx & \left(\Omega_{p}^{2} / 2\right) x-\Omega_{c o} v_{y} \\
& -\omega_{z} \Omega_{c w}[y \cos (2 \phi)-x \sin (2 \phi)] \\
d v_{y} / d t \approx & \left(\Omega_{p}^{2} / 2\right) y+\Omega_{c o} v_{x} \\
& -\omega_{z} \Omega_{c s}[x \cos (2 \phi)+y \sin (2 \phi)]
\end{aligned}
$$

and

$$
d v_{z} / d t \approx 0
$$

where

$$
\Omega_{p}^{2}=4 \pi n_{o} e^{2} / \gamma^{3} m, \Omega_{c o}=e B_{o} / \gamma m c, \Omega_{c w}=e B_{w} / \gamma m c
$$

and

$$
\omega_{z}=k_{w} v_{z}
$$

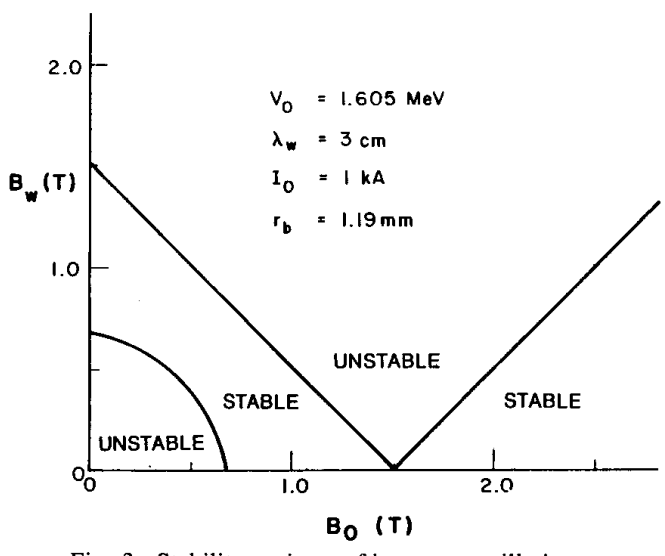

Fig. 3. Stability regimes of betatron oscillations.

The relativistic factor $\gamma=\left(1-v^{2} / c^{2}\right)^{-1 / 2}$ is constant in the static magnetic field. Note that $\Omega_{p}$ is the beam plasma frequency with a relativistic factor.

Following Levush et al. [1], we transform to the helical coordinate system defined by

$$
x_{1}=x \cos \phi+y \sin \phi
$$

and

$$
x_{2}=y \cos \phi-x \sin \phi .
$$

This coordinate system is rotating at half the pitch of the wiggler field helix. It leads to two-coupled, second-order differential equations with constant coefficients

$$
\begin{aligned}
& d^{2} x_{1} / d t^{2}+\left(\Omega_{c o}-2 \omega_{z}\right) d x_{2} / d t \\
& \quad+\left[\left(\Omega_{c o}-\omega_{z}\right) \omega_{z}-\Omega_{p}^{2} / 2\right] x_{1}+\omega_{z} \Omega_{c w} x_{2}=0
\end{aligned}
$$

and

$$
\begin{aligned}
& d^{2} x_{2} / d t^{2}-\left(\Omega_{c o}-2 \omega_{z}\right) d x_{1} / d t \\
& \quad+\left[\left(\Omega_{c o}-\omega_{z}\right) \omega_{z}-\Omega_{p}^{2} / 2\right] x_{2}+\omega_{z} \Omega_{c w} x_{1}=0 .
\end{aligned}
$$

\section{Stability}

The frequencies of the betatron oscillations with space charge are found by assuming a solution of the form $\exp (-i \omega t)$. One finds

$$
\begin{aligned}
\omega^{2}= & \Omega_{c o}^{2} / 2+\omega_{z}^{2}-\Omega_{c o} \omega_{z}-\Omega_{p}^{2} / 2 \\
& \pm\left[\Omega_{c w}^{2} \omega_{z}^{2}+\left(\Omega_{c o}^{2} / 4-\Omega_{p}^{2} / 2\right)\left(\Omega_{c o}-\omega_{z}\right)^{2}\right]^{1 / 2}
\end{aligned}
$$

so that the betatron oscillations in the unneutralized beam are unstable if

$$
\omega_{z}^{2} \Omega_{c w}^{2} \geq\left[\omega_{z}\left(\omega_{z}-\Omega_{c o}\right)+\Omega_{p}^{2} / 2\right]^{2}
$$

or

$$
\omega_{z}^{2} \Omega_{c w}^{2}<\left(\Omega_{c o}-2 \omega_{z}\right)^{2}\left(\Omega_{p}^{2} / 2-\Omega_{c o}^{2} / 4\right) .
$$

A plot of the stability boundaries as functions of $B_{w}$ and $B_{o}$ is shown in Fig. 3. The beam density determines the 
boundary in the lower left corner of the plot, while the upper boundary is insensitive to the beam density.

\section{E. Orbits}

The orbits of the electrons near the magnetic axis are found by using the Laplace transform. For the case with space charge, one finds

$$
\begin{aligned}
& x_{1}(t)=\frac{x_{1}(0)}{\left(\omega_{1}^{2}-\omega_{2}^{2}\right)}\left\{\left[\omega_{1}^{2} \cos \left(\omega_{1} t\right)-\omega_{2}^{2} \cos \left(\omega_{2} t\right)\right]\right. \\
& +\left[\omega_{z}\left(\omega_{z}-\Omega_{c o}\right)+\Omega_{p}^{2} / 2-\left(\Omega_{c o}-2 \omega_{z}\right)^{2}\right] \\
& \text { - }\left[\cos \left(\omega_{1} t\right)-\cos \left(\omega_{2} t\right)\right]-\Omega_{c w} \omega_{z}\left(\Omega_{c o}-2 \omega_{z}\right) \\
& \text { - } \left.\left[\sin \left(\omega_{1} t\right) / \omega_{1}-\sin \left(\omega_{2} t\right) / \omega_{2}\right]\right\} \\
& +\frac{x_{2}(0)}{\left(\omega_{1}^{2}-\omega_{2}^{2}\right)}\left\{\Omega_{c w} \omega_{z}\left[\cos \left(\omega_{1} t\right)-\cos \left(\omega_{2} t\right)\right]\right. \\
& +\left[\omega_{z}\left(\omega_{z}-\Omega_{c o}\right)+\Omega_{p}^{2} / 2\right]\left(\Omega_{c o}-2 \omega_{z}\right) \\
& \text { - } \left.\left[\sin \left(\omega_{1} t\right) / \omega_{1}-\sin \left(\omega_{2} t\right) / \omega_{2}\right]\right\} \\
& \cdot \frac{v_{1}(0)}{\left(\omega_{1}^{2}-\omega_{2}^{2}\right)}\left\{\left[\omega_{z}\left(\omega_{z}-\Omega_{c o}\right)+\Omega_{p}^{2} / 2\right]\right. \\
& \text { - }\left[\sin \left(\omega_{1} t\right) / \omega_{1}-\sin \left(\omega_{2} t\right) / \omega_{2}\right] \\
& \left.+\left[\omega_{1} \sin \left(\omega_{1} t\right)-\omega_{2} \sin \left(\omega_{2} t\right)\right]\right\} \\
& +\frac{v_{2}(0)}{\left(\omega_{1}^{2}-\omega_{2}^{2}\right)}\left\{\Omega _ { c w } \omega _ { z } \left[\sin \left(\omega_{1} t\right) / \omega_{1}\right.\right. \\
& \left.-\sin \left(\omega_{2} t\right) / \omega_{2}\right]+\left(\Omega_{c o}-2 \omega_{z}\right) \\
& \text { - } \left.\left[\cos \left(\omega_{1} t\right)-\cos \left(\omega_{2} t\right)\right]\right\} \\
& x_{2}(t)=\frac{x_{2}(0)}{\left(\omega_{1}^{2}-\omega_{2}^{2}\right)}\left\{\left[\omega_{1}^{2} \cos \left(\omega_{1} t\right)-\omega_{2}^{2} \cos \left(\omega_{2} t\right)\right]\right. \\
& +\left[\omega_{z}\left(\omega_{z}-\Omega_{c o}\right)+\Omega_{p}^{2} / 2-\left(\Omega_{c o}-2 \omega_{z}\right)^{2}\right] \\
& \text { - }\left[\cos \left(\omega_{1} t\right)-\cos \left(\omega_{2} t\right)\right] \\
& +\Omega_{c w} \omega_{z}\left(\Omega_{c o}-2 \omega_{z}\right) \\
& \text { - } \left.\left[\sin \left(\omega_{1} t\right) / \omega_{1}-\sin \left(\omega_{2} t\right) / \omega_{2}\right]\right\} \\
& +\frac{x_{1}(0)}{\left(\omega_{1}^{2}-\omega_{2}^{2}\right)}\left\{\Omega_{c w} \omega_{z}\left[\cos \left(\omega_{1} t\right)-\cos \left(\omega_{2} t\right)\right]\right. \\
& -\left[\omega_{z}\left(\omega_{z}-\Omega_{c o}\right)+\Omega_{p}^{2} / 2\right]\left(\Omega_{c o}-2 \omega_{z}\right) \\
& \text { - } \left.\left[\sin \left(\omega_{1} t\right) / \omega_{1}-\sin \left(\omega_{2} t\right) / \omega_{2}\right]\right\} \\
& +\frac{v_{2}(0)}{\left(\omega_{1}^{2}-\omega_{2}^{2}\right)}\left\{\left[\omega_{z}\left(\omega_{z}-\Omega_{c o}\right)+\Omega_{p}^{2} / 2\right]\right. \\
& \text { - }\left[\sin \left(\omega_{1} t\right) / \omega_{1}-\sin \left(\omega_{2} t\right) / \omega_{2}\right] \\
& \left.+\left[\omega_{1} \sin \left(\omega_{1} t\right)-\omega_{2} \sin \left(\omega_{2} t\right)\right]\right\} \\
& +\frac{v_{1}(0)}{\left(\omega_{1}^{2}-\omega_{2}^{2}\right)}\left\{\Omega _ { c w } \omega _ { z } \left[\sin \left(\omega_{1} t\right) / \omega_{1}\right.\right. \\
& \left.-\sin \left(\omega_{2} t\right) / \omega_{2}\right] \\
& \left.-\left(\Omega_{c o}-2 \omega_{z}\right)\left[\cos \left(\omega_{1} t\right)-\cos \left(\omega_{2} t\right)\right]\right\}
\end{aligned}
$$

$v_{1}(t)=\frac{v_{1}(0)}{\left(\omega_{1}^{2}-\omega_{2}^{2}\right)}\left\{\left[\omega_{1}^{2} \cos \left(\omega_{1} t\right)-\omega_{2}^{2} \cos \left(\omega_{2} t\right)\right]\right.$

$$
+\left[\omega_{z}\left(\omega_{z}-\Omega_{c o}\right)+\Omega_{p}^{2} / 2\right]
$$$$
\left.\cdot\left[\cos \left(\omega_{1} t\right)-\cos \left(\omega_{2} t\right)\right]\right\}
$$$$
+\frac{v_{2}(0)}{\left(\omega_{1}^{2}-\omega_{2}^{2}\right)}\left\{\Omega_{c w} \omega_{z}\left[\cos \left(\omega_{1} t\right)-\cos \left(\omega_{2} t\right)\right]\right.
$$$$
\left.-\left(\Omega_{c o}-2 \omega_{z}\right)\left[\omega_{1} \sin \left(\omega_{1} t\right)-\omega_{2} \sin \left(\omega_{2} t\right)\right]\right\}
$$$$
+\frac{x_{1}(0)}{\left(\omega_{1}^{2}-\omega_{2}^{2}\right)}\left\{-\left[\omega_{1}^{3} \sin \left(\omega_{1} t\right)-\omega_{2}^{3} \sin \left(\omega_{2} t\right)\right]\right.
$$$$
-\left[\omega_{z}\left(\omega_{z}-\Omega_{c o}\right)+\Omega_{p}^{2} / 2-\left(\Omega_{c o}-2 \omega_{z}\right)^{2}\right]
$$

- $\left[\omega_{1} \sin \left(\omega_{1} t\right)-\omega_{2} \sin \left(\omega_{2} t\right)\right]$

$\left.-\Omega_{c w} \omega_{z}\left(\Omega_{c o}-2 \omega_{z}\right)\left[\cos \left(\omega_{1} t\right)-\cos \left(\omega_{2} t\right)\right]\right\}$

$+\frac{x_{2}(0)}{\left(\omega_{1}^{2}-\omega_{2}^{2}\right)}\left\{-\Omega_{c w} \omega_{z}\left[\omega_{1} \sin \left(\omega_{1} t\right)\right.\right.$

$\left.-\omega_{2} \sin \left(\omega_{2} t\right)\right]$

$+\left[\omega_{z}\left(\omega_{z}-\Omega_{c o}\right)+\Omega_{p}^{2} / 2\right]\left(\Omega_{c o}-2 \omega_{z}\right)$

$\left.\cdot\left[\cos \left(\omega_{1} t\right)-\cos \left(\omega_{2} t\right)\right]\right\}$

$v_{2}(t)=\frac{v_{2}(0)}{\left(\omega_{1}^{2}-\omega_{2}^{2}\right)}\left\{\left[\omega_{1}^{2} \cos \left(\omega_{1} t\right)-\omega_{2}^{2} \cos \left(\omega_{2} t\right)\right]\right.$

$+\left[\omega_{z}\left(\omega_{z}-\Omega_{c o}\right)+\Omega_{p}^{2} / 2\right]$

- $\left.\left[\cos \left(\omega_{1} t\right)-\cos \left(\omega_{2} t\right)\right]\right\}$

$+\frac{v_{1}(0)}{\left(\omega_{1}^{2}-\omega_{2}^{2}\right)}\left\{\Omega_{c w} \omega_{z}\left[\cos \left(\omega_{1} t\right)-\cos \left(\omega_{2} t\right)\right]\right.$

$\left.+\left(\Omega_{c o}-2 \omega_{z}\right)\left[\omega_{1} \sin \left(\omega_{1} t\right)-\omega_{2} \sin \left(\omega_{2} t\right)\right]\right\}$

$+\frac{x_{2}(0)}{\left(\omega_{1}^{2}-\omega_{2}^{2}\right)}\left\{-\left[\omega_{1}^{3} \sin \left(\omega_{1} t\right)-\omega_{2}^{3} \sin \left(\omega_{2} t\right)\right]\right.$

$-\left[\omega_{z}\left(\omega_{z}-\Omega_{c o}\right)+\Omega_{p}^{2} / 2-\left(\Omega_{c o}-2 \omega_{z}\right)^{2}\right]$

- $\left[\omega_{1} \sin \left(\omega_{1} t\right)-\omega_{2} \sin \left(\omega_{2} t\right)\right]$

$\left.+\Omega_{c w} \omega_{z}\left(\Omega_{c o}-2 \omega_{z}\right)\left[\cos \left(\omega_{1} t\right)-\cos \left(\omega_{2} t\right)\right]\right\}$

$+\frac{x_{1}(0)}{\left(\omega_{1}^{2}-\omega_{2}^{2}\right)}\left\{-\Omega_{c w} \omega_{z}\left[\omega_{1} \sin \left(\omega_{1} t\right)\right.\right.$

$\left.-\omega_{2} \sin \left(\omega_{2} t\right)\right]$

$-\left[\omega_{z}\left(\omega_{z}-\Omega_{c o}\right)+\Omega_{p}^{2} / 2\right]\left(\Omega_{c o}-2 \omega_{z}\right)$

$\left.\cdot\left[\cos \left(\omega_{1} t\right)-\cos \left(\omega_{2} t\right)\right]\right\}$.

The frequencies $\omega_{1}$ and $\omega_{2}$ are the two betatron frequencies. The velocities $v_{1}$ and $v_{2}$ in the helical system are

$$
v_{1}=v_{x} \cos \phi+v_{y} \sin \phi+\omega_{z}[y \cos \phi-x \sin \phi]
$$

and

$v_{2}=v_{y} \cos \phi-v_{x} \sin \phi-\omega_{z}[x \cos \phi+y \sin \phi]$. 


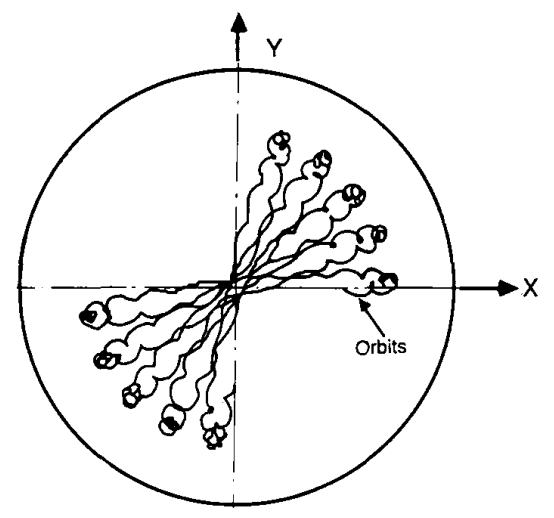

$\mathrm{B}_{\mathrm{w}}=0.305 \mathrm{~T}$

$\mathrm{B}_{0}=0$

$\mathrm{rg}_{\mathrm{g}}=6.59 \mathrm{~mm}$

$V_{b}=270 \mathrm{keV}$

Fig. 4. Betatron orbit with a small radius expansion.

The initial values of the coordinates and velocities are

$$
\begin{aligned}
& x_{1}(0)=x(0), x_{2}(0)=y(0), \\
& v_{1}(0)=v_{x}(0)+\omega_{z} y(0),
\end{aligned}
$$

and

$$
v_{2}(0)=v_{y}(0)-\omega_{z} x(0) .
$$

A polar plot of the orbit of a representative electron is shown in Fig. 4. This is one of the cases simulated by the nonlinear code and discussed in the next section. The parameters are $V_{b}=270 \mathrm{keV}, B_{o}=0, B_{w}\left(r_{g}\right)=0.0753 \mathrm{~T}$, and $r_{g}=0.659 \mathrm{~cm}$. The focusing effect of the quadrupole field is clearly shown. The electron oscillates through the magnetic axis five times while traveling $1.6 \mathrm{~m}$ along the axis. There are four betatron frequencies at $\omega_{1} \pm \omega_{z}$ and $\omega_{2} \pm \omega_{z}$. In this example these frequencies are $660 \mathrm{MHz}$, $725 \mathrm{MHz}, 14.4 \mathrm{GHz}$, and $15.8 \mathrm{GHz}$. The electron oscillates through the axis and slowly processes around the azimuth at a rate controlled by the two lower frequencies. The higher frequency wiggles are superimposed on this motion.

The computer simulation does not include the space charge and pinch effects. For comparison to the simulation the betatron frequencies are given by

$2 \omega^{2}=\left(\Omega_{c o}-\omega_{z}\right)^{2}+\omega_{z}^{2} \pm\left[4 \omega_{z}^{2} \Omega_{c w}^{2}+\left(\Omega_{c o}^{2}-2 \omega_{z}\right)^{2}\right]^{1 / 2}$

and the condition for instability is

$$
\Omega_{c w}^{2}>\left(\Omega_{c o}-\omega_{z}\right)^{2} \text {. }
$$

These linearized results are qualitatively confirmed by the nonlinear simulation.

Other beam instabilities may also play an important role. We find that annular beams produce the highest efficiency for this FEL configuration. In the presence of a finite guide field, an annular electron beam is vulnerable to instabilities caused by velocity shear produced by the space charge in the beam. For the velocity shear in the azimuthal rotation, this instability is known as the diocotron instability [10]. Recently, Lau [11] has examined this problem for a moderate current $(20 \mathrm{kA})$, large radius $(6.5 \mathrm{~cm})$ but thin annular beam which is being proposed as a driver for a wakefield accelerator, finding the spatial growth rate to be relatively small. We shall carry out a similar analysis for the case of a weak guide field and a strong helical quadrupole field. From Lau's results, we do not expect to diocotron instability to be a significant factor.

\section{F. The Frequency for Maximum Gain}

We find that the $\mathrm{TE}_{21}$ mode is amplified preferentially, since the electric fields of this mode match the quadrupole structure of the wiggler fields, which means that the field structure matches the lowest order pattern of the perturbed velocity of the electrons in the wiggler field.

We also find there is a class of solutions where the phase difference between the electrons and the signal field is stationary [12], so that

$$
\omega=v_{z}\left(k+2 k_{w}\right) .
$$

Including the dispersion relation for the waveguide field

$$
\omega^{2}=k^{2} c^{2}+k_{2 n}^{2} c^{2} \text { with } J_{2}^{\prime}\left(k_{2 n} r_{g}\right)=0
$$

and eliminating the wave vector $k$ from these two equations one finds the frequencies

$$
\omega=2 k_{w} v_{z} \gamma^{2}\left\{1 \pm\left[\beta^{2}-\left(\frac{k_{2 n}}{2 \gamma k_{w}}\right)^{2}\right]^{1 / 2}\right\}
$$

where

$$
\beta=v_{z} / c \text { and } \gamma=\left(1-\beta^{2}\right)^{-1 / 2} .
$$

The condition that the two curves are tangent yields

$$
\gamma=\left[1+\left(\frac{k_{2 n}}{2 k_{w}}\right)^{2}\right]^{1 / 2}
$$

and the frequency

$$
\omega=k_{2 n} c\left[1+\left(\frac{k_{2 n}}{2 k_{w}}\right)^{2}\right]^{1 / 2} .
$$

This equation predicts the frequency of maximum gain obtained from the computer simulation rather accurately. However, the bandwidth is very large.

III. Simulation of Nonlinear fEL Gain, EFFICIENCY, AND BANDWIDTH FOR A QUADRUPOLE WIGGLER IN THE 30-300 GHz RANGE

We have carried out simulations of the gain, efficiency, and bandwidth for 300 and $33 \mathrm{GHz}$ FEL's with a quadrupole wiggler. As previously mentioned, we have obtained a three-dimensional nonlinear code [2] from Ganguly and Freund of the Naval Research Laboratory. It 
treats waveguide modes in the Compton regime and has been used to analyze dipole helical wigglers and guide fields for FEL designs which have been compared with experimental helical dipole measurements at M.I.T. [5], [6] with good agreement. We have modified the code to treat quadrupole wiggler fields through changes in the wiggler field expression, adiabatic entries, and consequent modifications of the relativistic equations of motion.

For an FEL with a quadrupole wiggler and an axial guide field, the total magnetic field components of (2) are used for the nonlinear simulation. The characteristics of the quadrupole wiggler fields, shown in Fig. 2, are twofold: one is the null field at the center and the other is the rapid increase in the transverse dimension. This type of quadrupole wiggler has been shown to have strong focusing effects [1]. The adiabatic injection of the electron beam is modeled by allowing the wiggler amplitude to increase adiabatically over $N_{w}$ wiggler periods:

$$
B_{w(z)}= \begin{cases}B_{w o} \sin ^{2}\left(k_{w} z / 4 N_{w}\right) & 0 \leq z_{w} \leq N_{w} \lambda . \\ B_{w o} & N_{w} \lambda_{w} \leq z\end{cases}
$$

The code at present neglects space-charge effects. The vector potential of the radiation field can be expanded in terms of the eigenmodes of the vacuum waveguide provided that the space-charge effect is negligible. The waveguide modes can be calculated from the magnetic vector potential $\delta \vec{A}$ and Maxwell's equations as

$$
\delta \vec{E}=-\frac{1}{c} \frac{\partial}{\partial t} \delta \vec{A}, \text { and } \delta \vec{B}=\nabla \times \delta \vec{A}
$$

where

$$
\begin{aligned}
\delta \vec{A}(\vec{x}, t)= & \sum_{\substack{n=1 \\
l=0}}^{\infty} \delta A_{\ln }(z)\left\{\frac{l}{k_{\ln } r} J_{l}\left(k_{\ln } r\right) \hat{e}_{r} \sin \alpha_{l}\right. \\
& \left.+J_{l}^{\prime}\left(k_{\ln } r\right) \hat{e}_{\theta} \cos \alpha_{l}\right\} \\
& \text { for the } \mathrm{TE}_{\ln } \text { mode, or } \\
\delta \vec{A}(\vec{x}, t)= & \sum_{\substack{n=1 \\
l=0}}^{\infty} \delta A_{\ln }(s)\left\{J_{l}^{\prime}\left(k_{\ln } r\right) \cos \alpha_{l} \hat{e}_{r}\right. \\
& -\frac{l}{k_{\ln } r} J_{l}\left(k_{\ln } r\right) \sin \alpha \hat{e}_{\theta} \\
& \left.+\frac{k_{\ln }}{k} J_{l}\left(k_{\ln } r\right) \sin \alpha_{l} \hat{e}_{z}\right\} \\
& \text { for the } \mathrm{TM}_{\ln } \operatorname{mode}
\end{aligned}
$$

and

$$
\begin{aligned}
k(z) & =\text { wave number and } \\
\alpha_{l} & =\int_{0}^{z} d z^{\prime} k\left(z^{\prime}\right)+l \theta-\omega t .
\end{aligned}
$$

In the above equations $\delta \vec{A}(z)$ and $k(z)$ are slowly-varying functions of $z$ such that

$$
\begin{aligned}
\frac{d}{d z} \ln \left[\delta A_{\ln }(z)\right] & \ll k \\
\frac{d}{d z} \ln [k(z)] & \ll k
\end{aligned}
$$

The waveguide modes are described by

$$
\begin{array}{ll}
k_{\mathrm{ln}}=\frac{x_{\mathrm{ln}}^{\prime}}{r_{g}} & \text { for the } \mathrm{TE}_{\mathrm{ln}} \text { mode with } J_{l}^{\prime}\left(x_{\mathrm{ln}}^{\prime}\right)=0 \\
k_{\mathrm{ln}}=\frac{x_{\mathrm{ln}}}{r_{g}} & \text { for the } \mathrm{TM}_{\mathrm{ln}} \text { mode with } J_{l}\left(x_{\mathrm{ln}}\right)=0
\end{array}
$$

and $J_{l}\left(k_{\mathrm{ln}} r\right)$ and $J_{l}^{\prime}\left(k_{\mathrm{ln}} r\right)$ represent the lth-order Bessel function of the first kind and its derivative.

The derivation of the dynamic equations for both the TE and TM modes is formally similar to that by Ganguly and Freund [2] except the appropriate choice of the rotating coordinating system which rotates at the same pace as the helical quadrupole wiggler fields.

$$
\begin{aligned}
& \hat{e}_{1}=\hat{x} \cos \left(2 k_{w} z\right)+\hat{y} \sin \left(2 k_{w} z\right) \\
& \hat{e}_{2}=-\hat{x} \sin \left(2 k_{w} z\right)+\hat{y} \cos \left(2 k_{w} z\right) .
\end{aligned}
$$

The source current is described by a summation of all the electrons' motion as

$$
\begin{aligned}
\delta \vec{J}(\vec{x}, t)= & -e n_{b} v_{z 0} \int_{A_{g}} \int d x_{0} d y_{0} \sigma_{\perp}\left(x_{0}, y_{0}\right) \\
& \times \int_{-T / 2}^{T / 2} d t_{0} \sigma_{\|}\left(t_{0}\right) \vec{v}\left(z ; x_{0}, y_{0}, t_{0}\right) \\
& \cdot \frac{\delta\left(t-\tau\left(z ; x_{0}, y_{0}, t_{0}\right)\right)}{\left|v_{z}\left(z ; x_{0}, y_{0}, t_{0}\right)\right|}
\end{aligned}
$$

where the subscripts 0 denote initial conditions. Thus, we find the equations that follow. For the $\mathrm{TE}_{\mathrm{ln}}$ mode

$$
\begin{array}{r}
\frac{d^{2}}{d z^{2}} \delta a_{\ln }+\left[\frac{\omega^{2}}{c^{2}}-k^{2}-k_{\ln }^{2}\right] \delta a_{\ln } \\
=\frac{\omega_{b}^{2}}{c^{2}} \beta_{z 0} H_{\ln }\left\langle\frac{v_{1} T_{l}^{(+)}+v_{2} W_{l}^{(+)}}{\left|v_{z}\right|}\right\rangle \\
2 k^{1 / 2} \frac{d}{d z}\left(k^{1 / 2} \delta a_{\ln }\right) \\
=\frac{\omega_{b}^{2}}{c^{2}} \beta_{z 0} H_{\ln }\left\langle\frac{v_{1} W_{l}^{(-)}-v_{2} T_{l}^{(-)}}{\left|v_{z}\right|}\right\rangle
\end{array}
$$


and for the $\mathrm{TM}_{\mathrm{In}}$ mode

$$
\begin{aligned}
& \frac{d^{2}}{d z^{2}} \delta a_{\ln }+\left[1+\frac{k_{\ln }^{2}}{k^{2}}\right]\left[\frac{\omega^{2}}{c^{2}}-k^{2}-k_{\ln }^{2}\right] \delta a_{\ln } \\
& =\frac{\omega_{b}^{2}}{c^{2}} \beta_{z o} H_{\ln }\left\langle\frac{v_{1} T_{l}^{(+)}+v_{2} W_{l}^{(+)}+2 \frac{k_{\ln }}{k} v_{z} J_{l}\left(k_{\ln } r\right) \sin \alpha_{l}}{\left|v_{z}\right|}\right\rangle \\
& 2\left(k+\frac{k_{\ln }^{2}}{k}\right)^{1 / 2} \frac{d}{d z}\left[\left(k+\frac{k_{\ln }^{2}}{k}\right)^{1 / 2} \delta a_{\ln }\right] \\
& =\frac{w_{b}^{2}}{c^{2}} \beta_{z o} H_{\ln }\left\langle\frac{v_{1} W_{l}^{(-)}-v_{2} T_{l}^{(-)}+2 \frac{k_{\ln }}{k} v_{z} J_{l}\left(k_{\ln } r\right) \cos \alpha_{l}}{\left|v_{z}\right|}\right\rangle
\end{aligned}
$$

where

$$
\delta a_{\ln } \equiv e \delta A_{\ln } / m c^{2}, \beta_{z 0} \equiv v_{z 0} / c, \omega_{b}^{2} \equiv 4 \pi e^{2} n_{b} / m
$$

and

$$
H_{\ln }= \begin{cases}\frac{\left(x_{\ln }^{\prime 2}\right)}{\left[\left(x_{\ln }^{\prime 2}\right)-l^{2}\right] J_{l}^{2}\left(x_{\ln }^{\prime}\right)} & \text { for the } \mathrm{TE}_{\mathrm{ln}} \text { mode } \\ {\left[J_{l}^{\prime}\left(\chi_{\mathrm{ln}}\right)\right]^{-2}} & \text { for the } \mathrm{TM}_{\mathrm{ln}} \text { mode. }\end{cases}
$$

Finally, the dynamic equations of the relativistic electrons can be derived from the Lorentz force equation in the presence of the quadrupole wiggler fields and electromagnetic fields. Since we consider the amplifier mode and neglect the space-charge potential, the equations become

$$
\frac{d \vec{p}}{d t}=-e \delta \vec{E}_{\ln }-e \frac{\vec{v}}{c} \times\left(\vec{B}_{o}+\vec{B}_{w}+\delta \vec{B}_{\ln }\right) .
$$

We transform to a coordinate system that is rotating in phase with the helical wiggler. The new momenta $\left(p_{1}\right.$, $p_{2}$ ) are given by

$$
p_{1}=p_{x} \cos \left(2 k_{w} z\right)+p_{y} \sin \left(2 k_{w} z\right)
$$

and

$$
p_{2}=p_{y} \cos \left(2 k_{w} z\right)-p_{x} \sin \left(2 k_{w} z\right) .
$$

Defining a new independent variable $z$, with $d z=v_{z} d t$ we find, for TE modes

$$
\begin{aligned}
v_{z} \frac{d}{d z} p_{1}= & -\left[\Omega_{c o}-2 k_{w} v_{z}+2 \Omega_{c w} M_{2}\right] p_{2}+\Omega_{c w} p_{z} M_{2} \\
& -\frac{1}{2} m c \delta a_{\ln }\left[\left(\omega-k v_{z}\right) W_{l}^{(-)}-2 k_{\ln } v_{2} J_{l}\left(k_{\ln } r\right)\right. \\
& \left.\cdot \cos \alpha_{l}-\Gamma_{\ln } v_{z} T_{l}^{(+)}\right] \\
v_{z} \frac{d}{d z} p_{2}= & {\left[\Omega_{c o}-2 k_{c w} v_{z}+2 \Omega_{c w} M_{0}\right] p_{1}-\Omega_{c w} p_{z} M_{1} } \\
& +\frac{1}{2} m c \delta a_{\ln }\left[\left(\omega-k v_{z}\right) T_{l}^{(-)}-2 k_{\ln } v_{1} J_{l}\left(k_{\ln } r\right)\right. \\
& \left.\cdot \cos \alpha_{l}+\Gamma_{\ln } v_{z} W_{l}^{(+)}\right]
\end{aligned}
$$

$$
\begin{aligned}
v_{z} \frac{d}{d z} p_{z}= & \Omega_{c w} p_{2} M_{1}-\Omega_{c w} p_{1} M_{2} \\
& -\frac{1}{2} m c \delta a_{\ln }\left[k\left(v_{1} W_{l}^{(-)}-v_{2} T_{l}^{(-)}\right)\right. \\
& \left.+\Gamma_{\ln }\left(v_{1} T_{l}^{(+)}+v_{2} W_{l}^{(+)}\right)\right] .
\end{aligned}
$$

For TM modes we find

$$
\begin{aligned}
v_{z} \frac{d p_{1}}{d z}= & -\left[\Omega_{c o}-2 k_{w} v_{z}+2 \Omega_{c w} M_{o}\right] p_{2}+\Omega_{c^{\prime} w} p_{z} M_{2} \\
& -\frac{1}{2} m c \delta a_{\ln }\left\{\left[\omega-\left(k+\frac{k_{\ln }^{2}}{k}\right) v_{z}\right] W_{l}^{(-)}\right. \\
& \left.-\Gamma_{\ln } v_{z} T_{l}^{(+)}\right\}
\end{aligned}
$$

$v_{z} \frac{d p_{2}}{d z}=-\left[\Omega_{c o}-2 k_{w} v_{z}+2 \Omega_{c w} M_{o}\right] p_{1}-\Omega_{c w} p_{z} M_{1}$

$$
\begin{aligned}
& +\frac{1}{2} m c \delta a_{\ln }\left\{\left[\omega-\left(k+\frac{k_{\ln }^{2}}{k}\right) v_{z}\right] T_{l}^{(-)}\right. \\
& \left.+\Gamma_{\ln } v_{z} W_{l}^{(+)}\right\}
\end{aligned}
$$

$v_{z} \frac{d p_{z}}{d z}=-\Omega_{c w} M_{1} p_{1}+\Omega_{c w} M_{1} p_{2}$

$$
\begin{aligned}
& -\frac{1}{2} m c \delta a_{\ln }\left[\left(k+\frac{k_{\ln }^{2}}{k}\right)\left(v_{1} W_{l}^{(-)}-v_{2} T_{l}^{(-)}\right)\right. \\
& +\Gamma_{\ln }\left(v_{1} T_{l}^{(+)}+v_{2} W_{l}^{(+)}\right) \\
& \left.+2 \omega \frac{k_{\ln }}{k} J_{l}\left(k_{\ln } r\right) \cos \alpha_{l}\right]
\end{aligned}
$$

where

$$
\begin{aligned}
& M_{0}=I_{2}(2 \lambda) \sin 2 \chi \\
& M_{1}=I_{1}(2 \lambda) \cos \theta+I_{3}(2 \lambda) \cos (4 \chi-\theta) \\
& M_{2}=-I_{1}(2 \lambda) \sin \theta+I_{3}(2 \lambda) \sin (4 \chi-\theta)
\end{aligned}
$$




$$
\begin{aligned}
T_{l}^{( \pm)} & = \begin{cases}F_{l}^{( \pm)} \sin \psi_{l}+G_{l}^{( \pm)} \cos \psi_{l} & \mathrm{TE}_{\mathrm{ln}} \text { mode } \\
F_{l}^{(\mp)} \cos \psi_{l}-G_{l}^{(\mp)} \sin \psi_{l} & \mathrm{TM}_{\mathrm{ln}} \text { mode }\end{cases} \\
W_{l}^{( \pm)} & = \begin{cases}F_{l}^{(\mp)} \cos \psi_{l}-G_{l}^{(\mp)} \sin \psi_{l} & \mathrm{TE}_{\mathrm{ln}} \text { mode } \\
-F_{l}^{( \pm)} \sin \psi_{l}-G_{l}^{( \pm)} \cos \psi_{l} & \mathrm{TM}_{\mathrm{ln}} \text { mode }\end{cases}
\end{aligned}
$$

with

$$
\begin{aligned}
\psi_{l} \equiv & \psi_{0}+\int_{0}^{z}\left(k+l k_{w}-\frac{\omega}{v_{z}}\right) d z^{\prime} \\
F_{l}^{( \pm)}= & J_{l-1}\left(k_{\ln } r\right) \cos ((l-2) \chi+\theta) \pm J_{l+1}\left(k_{\ln } r\right) \\
& \cdot \cos ((l+2) \chi-\theta) \\
G_{l}^{( \pm)}= & J_{l-1}\left(k_{\ln } r\right) \sin ((l-2) \chi+\theta) \pm J_{l+1}\left(k_{\ln } r\right) \\
& \cdot \sin ((l+2) \chi-\theta) .
\end{aligned}
$$

In addition, the electron coordinates obey

$$
\begin{aligned}
v_{z} \frac{d x}{d z} & =v_{1} \cos \left(2 k_{w} z\right)-v_{2} \sin \left(2 k_{w} z\right) \\
v_{z} \frac{d y}{d z} & =v_{1} \sin \left(2 k_{w} z\right)+v_{2} \cos \left(2 k_{w} z\right) \\
\frac{d \psi_{l}}{d z} & =k+l k_{w}-\frac{\omega}{v_{z}}
\end{aligned}
$$

Equations (17), (18), and (21)-(23) constitute the nonlinear and fully-relativistic 3-D dynamic equations governing the interacting evolution of the waveguide mode and the electrons. These equations are integrated with a fourthorder Runge-Kutta algorithm, and the integrations over the beam cross section in (17) are done with a Gaussian quadrature method. Typically, 1000 beam electrons are followed.

\section{Results of Nonlinear Simulation}

\section{A. General Results for Quadrupole Cases}

We have simulated the operation of this FEL configuration in two wavelength regimes, near $33 \mathrm{GHz}$ and near $300 \mathrm{GHz}$. The condition that the beam resonance curve and the waveguide dispersion curve be tangent, given by (10) and (11), predicts accurately the FEL parameters for maximum gain and efficiency. In these cases we have held the wiggler period fixed at $3 \mathrm{~cm}$, and have varied the beam voltage, beam current, waveguide radius, and wiggler field intensity to optimize the FEL performance. In these cases there is no axial magnetic field.

For an example in the $33 \mathrm{GHz}$ range, the tangential intersection condition requires $0.27 \mathrm{MeV}$ beam voltage and $0.66 \mathrm{~cm}$ waveguide radius for a quadrupole wiggler with a $3 \mathrm{~cm}$ period. The beam current is $500 \mathrm{~A}$, the wiggler field is $3.05 \mathrm{kG}$ at the wall, and an annular beam is used with constant current density between an inner radius of $0.33 \mathrm{~cm}$ and an outer radius of $0.52 \mathrm{~cm}$. Figs. 5 and 6 shows the moderate efficiency ( 9 percent) and the broad spectral response ( 40 percent bandwidth) that are attained by holding these parameters constant and varying the frequency. Fig. 7 shows the spatial evolution of the power

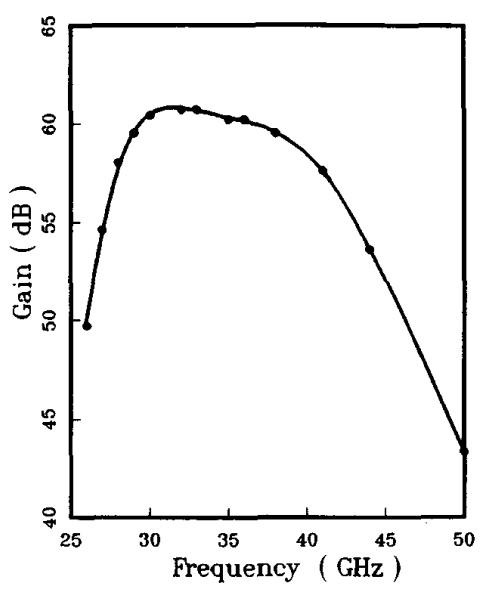

Fig. 5. Spectral response of gain for $\mathrm{TE}_{21}$ mode for the FEL with a quadrupole wiggler at $33 \mathrm{GHz}$. The parameters are shown in Fig. 7.

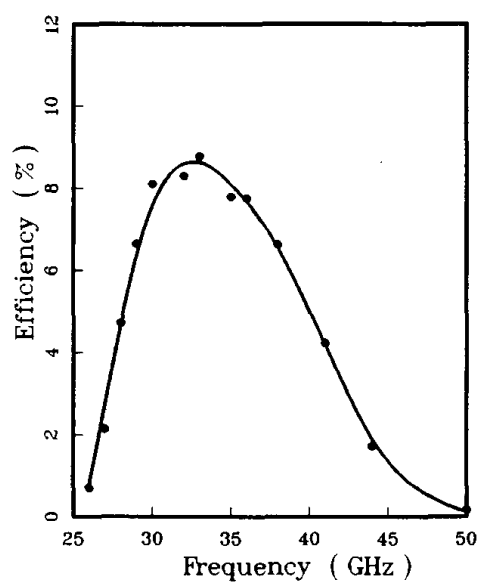

Fig. 6. Spectral response of efficiency for $\mathrm{TE}_{21}$ for the FEL with a quadrupole wiggler at $33 \mathrm{GHz}$. The parameters are shown in Fig. 7 .

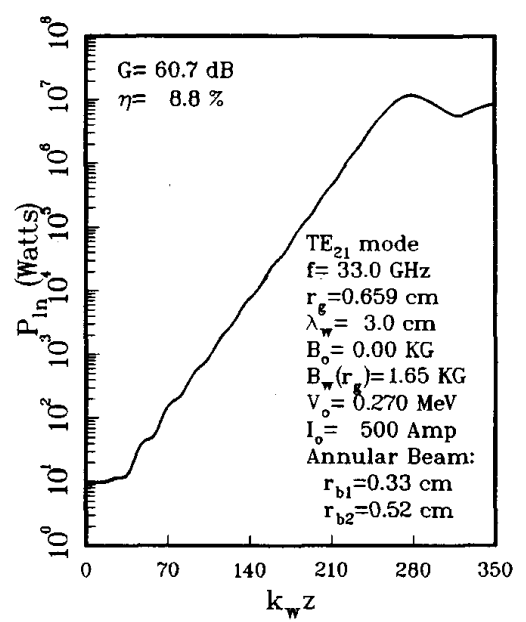

Fig. 7. Spatial evolution of $\mathrm{TE}_{21}$ mode for the FEL with a quadrupole wiggler at $33 \mathrm{GHz}$.

and the growth rate of the $\mathrm{TE}_{21}$ mode, the dominate mode in this case. It shows a $0.46 \mathrm{~dB} / \mathrm{cm}$ spatial gain. The rapid fluctuation of the growth rate is due to the transverse motion of the electrons traveling through the strongly-inho- 


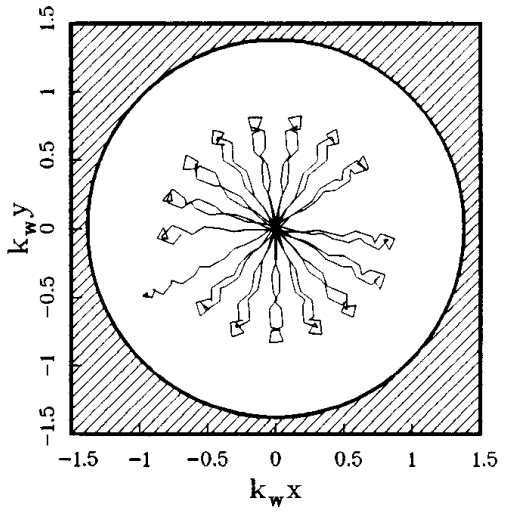

Fig. 8. Nonlinear betatron orbit of an electron for the FEL with a quad rupole wiggler at $33 \mathrm{GHz}$. The parameters are shown in Fig. 7.

mogeneous wiggler field. The transverse orbit of one of the electrons is shown in Fig. 8, in which the spiral-like trajectory at the lower-left quarter corresponds to the electron traveling through the adiabatic region and, consecutively, the figure eight trajectories are the betatron motion. Note that the electron is strongly focused due to the inhomogeneity of the transverse wiggler field components. These orbits qualitatively agree with the linearized orbits that are illustrated in Fig. 4 and the betatron frequencies agree to within 10 percent. The orbits of Fig. 9 are generated in a different manner; the positions in the transverse plane of 100 electrons are shown as the axial distance along the guide tube is increased. The electrons are initially distributed with uniform spacing in radius, and a constant azimuthal angle. The angles are the roots of the Legendre polynomials that are used in the Gaussian quadrature integration over the cross section of the electron beam. In this way the figure shows qualitatively the behavior of the electron beam profile.

To scale up to the $300 \mathrm{GHz}$ range, we increase the beam voltage to $1.6 \mathrm{MeV}$ and reduce the waveguide radius to $0.2 \mathrm{~cm}$ such that the tangential intersection conditions are still satisfied. Also, this FEL has a moderate efficiency ( 5 percent), broad spectral response (50 percent), and the spatial gain is $0.47 \mathrm{~dB} / \mathrm{cm}$. However, this extremelysmall waveguide size is difficult to realize. We can allow the beam resonance curve intersecting the waveguide dispersion curve at two frequencies with the upper one corresponding to the desired frequency. Keeping the same waveguide radius as in the $33 \mathrm{GHz}$ case, this criteria requires a larger beam voltage and beam current, and a stronger wiggler intensity. The simulation results in Figs. 10-12 show that the efficiency drops to 2 percent and the bandwidth decreases to 8 percent, but the spatial gain increases to $0.55 \mathrm{~dB} / \mathrm{cm}$, which is due to the larger beam current employed. Figs. 13 and 14 show the electron betatron orbits and bulk trajectories for the and $300 \mathrm{GHz}$ case. They are similar to the $33 \mathrm{GHz}$ case, except for the slightly different betatron frequencies. Again, the compression of the electron beam radius along the waveguide, shown in Fig. 13, is observed for the $300 \mathrm{GHz}$ case, due to the strong focusing effect of the quadrupole wiggler.

\section{B. Comparison of the Quadrupole and Dipole Helical Wigglers at $35 \mathrm{GHz}$}

The construction of a free-electron laser with a helical quadrupole wiggler is similar to an FEL with a helical dipole wiggler, in the millimeter wavelength range where both are expected to operate efficiently. In both cases the wiggler field is produced by currents in helical conductors that are wound on a machined mandrel that fits closely over the circular waveguide-guide tube. The dipole requires two wires with opposing currents (a bifilar wiggler), and the quadrupole requires four wires. In both cases the signal field is a circular waveguide mode that is circularly polarized, the $\mathrm{TE}_{11}$ mode for the dipole and the $\mathrm{TE}_{21}$ mode for the quadrupole. For the current experiments the frequency is low enough so that higher order modes are cut off, but at millimeter wavelengths many parasitic modes can propagate, and must be discriminated. Mode converters near the FEL output will be needed for particular applications, and mode transforming antenna structures will be needed to produce tight radiation patterns. These mode converters have been extensively developed for use with millimeter wave gyrotrons.

Our nonlinear computer simulations at $33 \mathrm{GHz}$ confirm that the FEL with an untapered helical quadrupole wiggler discriminates very efficiently against the higher order modes with high gain. This means that the gain at the design frequency for all modes other than the $\mathrm{TE}_{2 n}$ modes is essentially zero. We are now modifying the computer program to allow many modes to be amplified at the same time, to further study the problem of mode competition.

As we have already noted, the quadrupole wiggler field is zero on-axis and increases with the radius. For efficient conversion of beam power to radiation power, an annular electron beam is most efficient, while a solid beam is better for the dipole wiggler. The hollow beam may be used to advantage to discriminate between the $\mathrm{TE}_{2 n}$ modes. A thin annular electron beam may be tailored with a radius at a radial maximum of a particular $\mathrm{TE}_{2 n}$ mode, amplifying this single mode. This method is now used in gyrotron design.

Further comparisons of the FEL with a quadrupole and with a dipole helical wiggler are shown in Table I, with data from similar nonlinear simulations. The solid electron beam for the dipole wiggler and the annular beam for the quadrupole wiggler have comparable beam power density. The waveguide size is adjusted so that the interaction occurs over the same frequency range. The gains and efficiencies that are quoted are the peak values within the bandwidth.

The most striking difference between the two FEL configurations is the difference in beam voltage and beam current needed to produce the optimum gain and efficiency. The helical quadrupole FEL requires much lower beam voltage and much higher beam current than the di- 

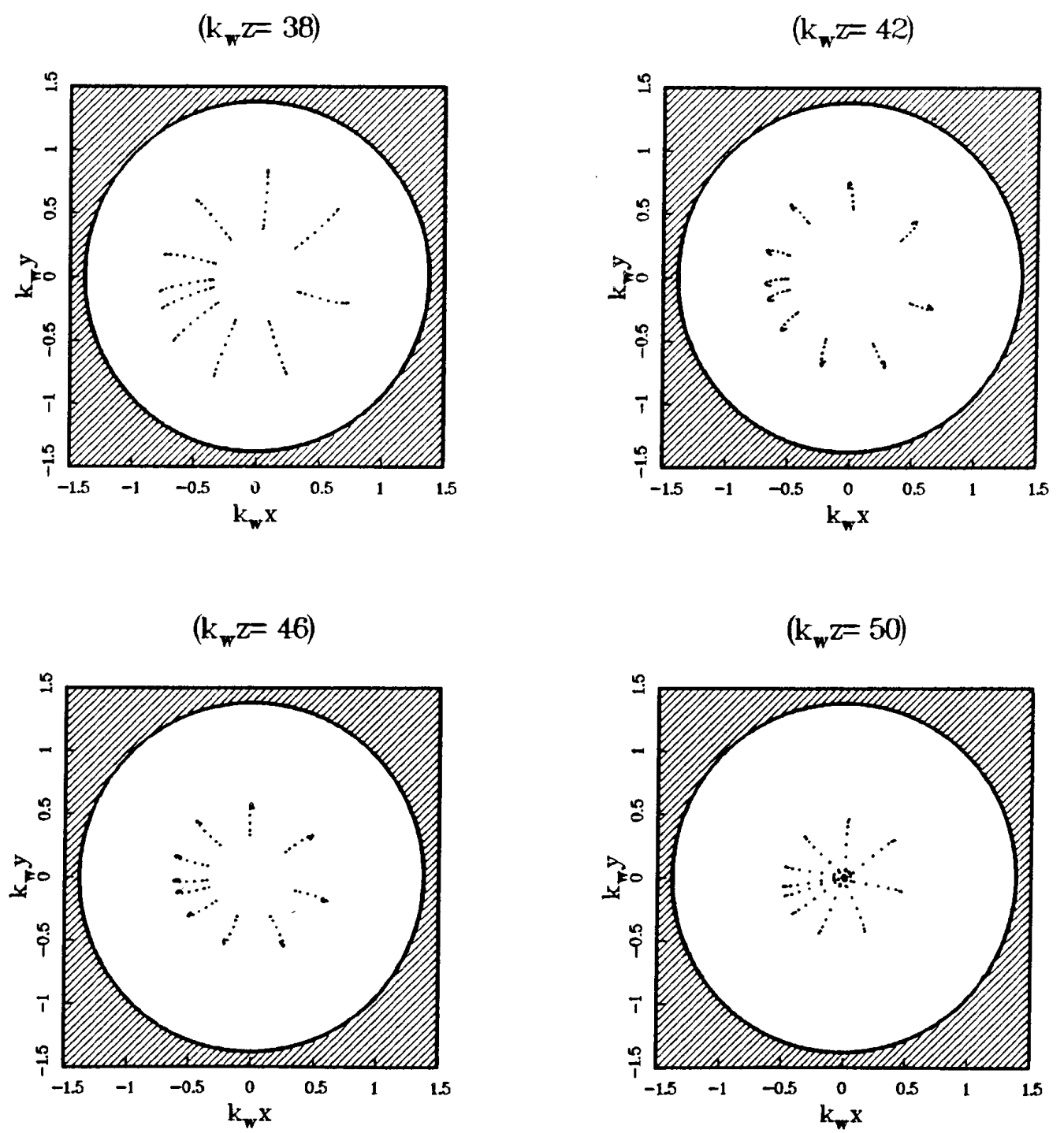

Fig. 9. Electron beam profiles for the FEL with a quadrupole wiggler at $33 \mathrm{GHz}$. The parameters are shown in Fig. 7.

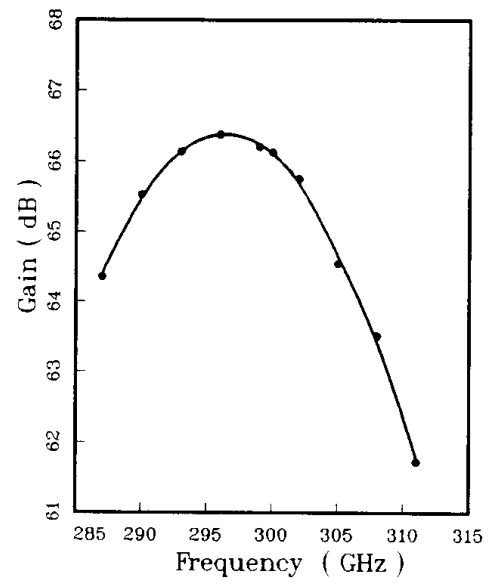

Fig. 10. Spectral response of gain for TE $\mathrm{T}_{21}$ mode for the FEL with a quadrupole wiggler at $300 \mathrm{GHz}$. The parameters are shown in Fig. 12.

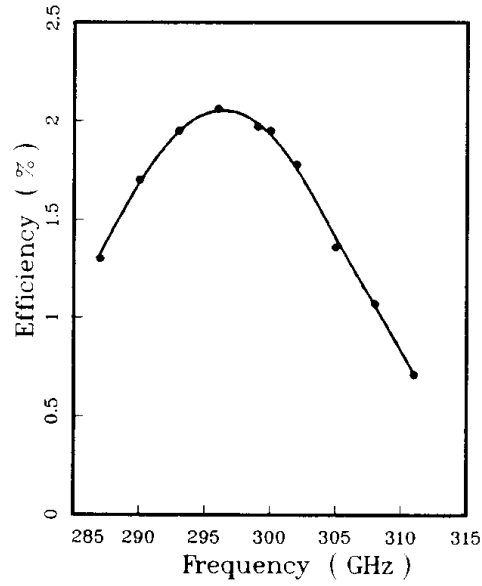

Fig. 11. Spectral response of efficiency for $\mathrm{TE}_{2 !}$ mode for the FEL with a quadrupole wiggler at $300 \mathrm{GHz}$. The parameters are shown in Fig. 12. 


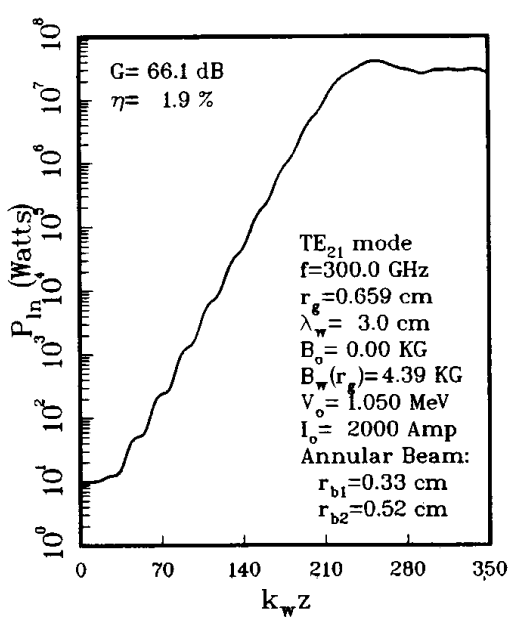

Fig. 12. Spatial evolution of $\mathrm{TE}_{21}$ mode for the FEL with a quadrupole wiggler at $300 \mathrm{GHz}$.

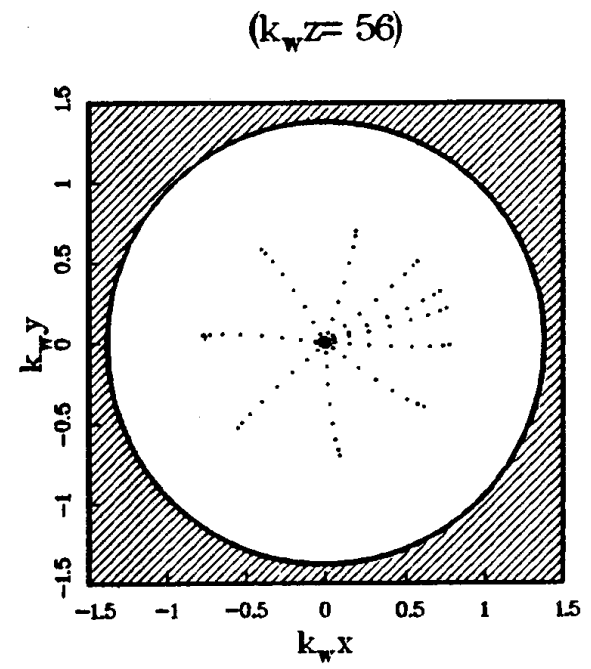

$\left(k_{w} z=64\right)$

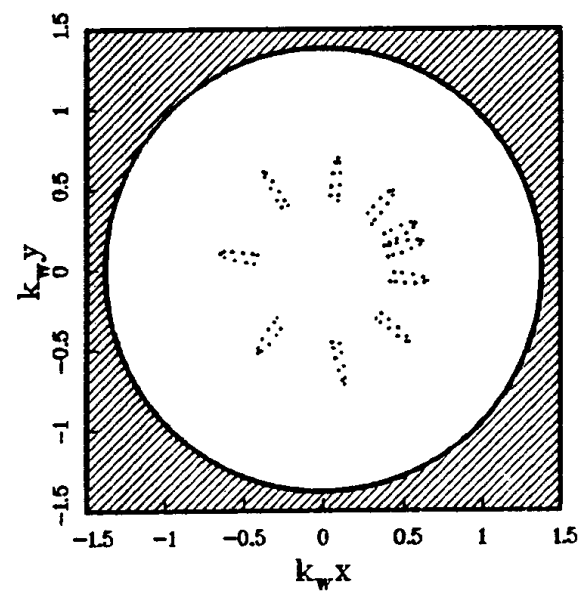

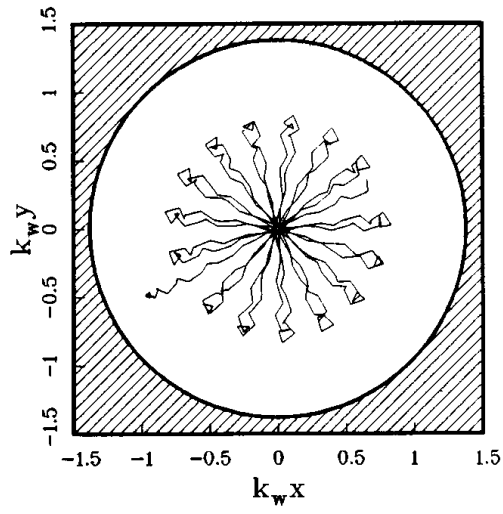

Fig. 13. Nonlinear betatron orbit of an electron for the FEL with a quadrupole wiggler at $300 \mathrm{GHz}$. The parameters are shown in Fig. 12.
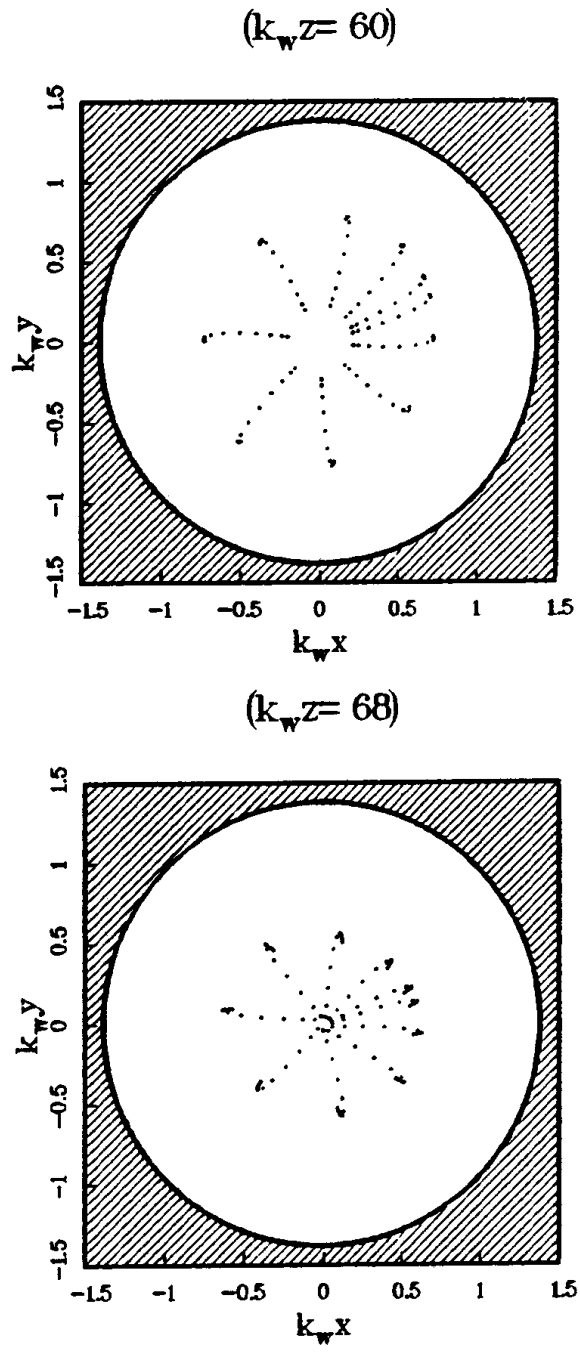

Fig. 14. Electron beam profiles for the FEL with a quadrupole wiggler at $300 \mathrm{GHz}$. The parameters are shown in Fig. 12. 
TABLE I

COMPaRISON OF Dipole and QuadruPOle Wigglers at $35 \mathrm{GHz}^{\text {a }}$

\begin{tabular}{|c|c|c|c|c|}
\hline & Parameter & Dipole & Quadrupole & Units \\
\hline Beam & $\begin{array}{l}V_{b} \\
I_{b} \\
r_{b 1} / r_{b 2} \\
P_{b} \\
P_{b} / A_{b}\end{array}$ & $\begin{array}{l}0.6 \\
50.0 \\
0.0 / 0.2 \\
30 \\
238\end{array}$ & $\begin{array}{l}0.27 \\
500 \\
0.33 / 0.52 \\
135 \\
266\end{array}$ & $\begin{array}{l}\mathrm{MeV} \\
\mathrm{A} \\
\mathrm{cm} / \mathrm{cm} \\
\mathrm{MW} \\
\mathrm{MW} / \mathrm{cm}^{2}\end{array}$ \\
\hline Wiggler & $\begin{array}{l}\lambda_{w} \\
B_{w} \\
\left\langle B_{w}\right\rangle \text { over Beam } \\
B_{o}\end{array}$ & $\begin{array}{l}3.0 \\
1.0 \\
1.0 \\
0\end{array}$ & $\begin{array}{l}3.0 \\
0.75 \\
0.54 \\
0\end{array}$ & $\begin{array}{l}\mathrm{cm} \\
\mathrm{kG} \\
\mathrm{kG} \\
\mathrm{kG}\end{array}$ \\
\hline Waveguide & $\begin{array}{l}f_{o} \\
r_{g} \\
L \\
\text { mode }\end{array}$ & $\begin{array}{c}35 \\
0.5 \\
124 \\
\mathrm{TE}_{11}^{0}\end{array}$ & $\begin{array}{l}33 \\
0.66 \\
133 \\
T E_{21}^{0}\end{array}$ & $\begin{array}{l}\mathrm{GHz} \\
\mathrm{cm} \\
\mathrm{cm}\end{array}$ \\
\hline $\begin{array}{l}\text { Efficiency } \\
\text { Gain } \\
\text { Bandwidth } \\
\text { Normalized } \\
\text { Normalized }\end{array}$ & $\begin{array}{l}\eta \\
G \\
\mathrm{BW} \\
\langle\eta\rangle \\
\langle G\rangle\end{array}$ & $\begin{array}{l}15.4 \\
56.4 \\
56 \\
0.12 \\
0.46\end{array}$ & $\begin{array}{l}8.8 \\
60.7 \\
40 \\
0.12 \\
0.85\end{array}$ & $\begin{array}{l}\text { percent } \\
\mathrm{dB} \\
\text { percent } \\
\text { percent }(\mathrm{kG} \cdot \mathrm{cm})^{-1} \\
\mathrm{~dB}(\mathrm{kG} \cdot \mathrm{cm})^{-1}\end{array}$ \\
\hline
\end{tabular}

a $\langle A\rangle=A /\left\langle B_{n}\right\rangle L$

pole configuration. The lower voltage and higher current is easier to produce with currently available electron beam accelerators, and provides a better "impedance" match, and a lower voltage standoff requirement.

Each of the FEL amplifier configurations has a very wide bandwidth, high gain, and moderate efficiency. In each case the efficiency may be improved by optimizing the taper of the wiggler, which we will carry out in future research.

\section{Summary}

We have examined the characteristics of a Compton regime quadrupole magnetic wiggler for a FEL amplifier in the millimeter frequency range $30-300 \mathrm{GHz}$. We find that an annular beam with a $\mathrm{TE}_{21}$ circular waveguide mode propagating yields the optimum gain and efficiency. The particle orbit trajectories including betatron oscillations and quadrupole focusing have been examined. Optimum efficiencies (9-2 percent) for the untapered quadrupole occur for lower beam voltages, larger guide radii, and shorter total wiggler lengths than for a comparable dipole case. Higher currents and substantial wiggler field intensities are required to optimize the gain and efficiency for the quadrupole. The effects of a tapered wiggler mode competition, and space-charge effects will be examined in future work.

\section{REFERENCES}

[1] B. Levush et al., "A free electron laser with a rotating quadrupole wiggler," Phys. Fluids, vol. 28, pp. 2273-2281, 1985

[2] A. K. Ganguly and H. P. Freund, "Nonlinear analysis of free-electron-laser amplifiers in three dimensions," Phys. Rev., vol. A32, pp. 2275-2286, 1985.

[3] J. Scharer. O. Eldridge, S. F. Chang, and N. Lam, “Nonlinear analysis and simulation of a millimeter range free electron laser with hel- ical quadrupole wiggler," in Proc. 9th Int. Free Electron Las"r Conf., Sept. 14-18, 1987, Williamsburg, VA, to be published.

[4] J. Fajans, J. S. Wurtele et al. " Nonlinear power saturation and phase (wave refractive index) in a collective free-electron laser amplifier, Phys. Rev. Lett., vol. 57, pp. 579-582, 1986.

[5] J. Fajans, G. Bekefi, Y. Z. Yin, and B. Lax, "Microwave studies of a tunable free-electron laser in combined axial and wiggler magnetic fields,' Phys. Fluids, vol. 28, pp. 1995-2006. 1985.

[6] J. Fajans, D. A. Kirkpatrick, and G. Bekefi, "Off-axis electron orbits in relativistic helical wiggler for free-electron-laser applications.' Phys. Rev., vol. A32, pp. 3488-3453, 1985.

[7] D. E. Pershing, R. H. Jackson, and R. K. Parker, IOth Int. Conf. Infared, Millimeter Waves, Orlando, FL, Dec. 9-13, 1985

[8] B. Levush, T. M. Antonsen, Jr. and W. M. Mannheimer, "Spontaneous radiation of an electron beam in a free-electron laser with a quadrupole wiggler," J. Appl. Phys., vol. 60, pp. 1584-1590, 1986.

191 T. M. Antonsen, Jr., and B. Levush, "Nonlinear theory of a quadrupole free electron laser,' Univ. Maryland Rep. 1987.

[10] O. Buneman, R. H. Levy, and L. M. Linson, "Stability of crossedfield electron beams,"J. Appl. Phys, , vol. 37, pp. 3203-3222, 1966.

[11] Y. Y. Lau, "Some design considerations on using modulatec intense annular beams for Wakefield acceleration." NRL Memo. Rep. 5981, 1987

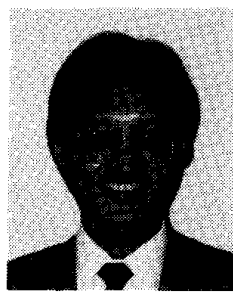

Sheng-Fuh R. Chang was born in Taiwan, Republic of China, in 1960 . He received the B.S and M.S. degrees in communication engineering from the National Chiao-Tung University in 1982 and 1984 , respectively.

Currently, he is working towards the Pr. D. degree in electrical and computer engineering at the University of Wisconsin, Madison. His research interests are in free-electron lasers, plasma waves, and microstrip circuits. 
Owen C. Eldridge was born in Crossville, TN, in 1932. He received the B.S. degree in engineering physics from the University of Tennessee in 1953 and the Ph.D. degree in physics from the University of California, Berkeley, in 1960.

He has performed research in plasma physics and engineering at General Atomic and at Oak Ridge National Laboratory, concentrating on nonlinear waves and instabilities in plasmas, and on the RF heating of plasmas. $\mathrm{He}$ has taught physics and engineering at the University of Tennessee, Knoxville, at the City University of New York, Hunter College, New York, and at the University of Wisconsin, Madison. He is currently doing free-electron laser research and teaching with the Department of Electrical and Computer Engineering, University of Wisconsin, Madison.

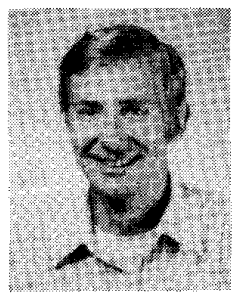

John E. Scharer was born in Monroe, WI, in 1939. He received the B.S., M.S., and Ph.D. degrees in electrical engineering from the University of California, Berkeley, in plasma physics.

$\mathrm{He}$ is a Professor with the Department of Electrical and Computer Engineering, University of Wisconsin, Madison. He has spent research sabatticals at the CEA Fontenay-aux-Roses, France, in 1970 and at the JET tokamak at Culham, England in 1983 . He has been active with graduate students and scientists in theoretical, computational, and experimental research in the area of linear and nonlinear plasm waves and heating of fusion plasmas and lasers. He has also taught and recently started research in the area of free-electron lasers and coherent sources of radiation.

Dr. Scharer is a member of Tau Beta Pi, the American Physical Society, and an affiliate member of the IEEE. 